\title{
A NOTE UPON THE FARADIC STIMULATION OF THE POSTCENTRAL GYRUS IN CONSCIOUS PATIENTS. ${ }^{1}$
}

\author{
BI HARVEX CUSHING, M.D.
}

Associate Professor of Surgery, Tho Johns Hopkins Hospital, Baltimore.

ONE of the most important steps in our progress toward a more exact localization of function in the cerebral cortex was taken when, by a method of unipolar faradization, Sherrington and Grünbaum accurately delineated the confines of the so-called motor area. Their circumscription of this electrically excitable strip of cortex in the ape to the anterior: wall of the fissura centralis (Rolandi) and to the adjoining surface of the precentral gyrus, has received confirmation through the more recent studies of myelinization, and through investigations into the finer histology of the cortex, which have shown that this area corresponds with the distribution of the Betz cells. Sherrington and Grünbaum's observations on the anthropoid brain, furthermore, have since been confirmed for man by Krause and Frazier ; and I have had the occasion myself to stimulate the cortex of more than fifty anresthetized patients with results in exact accord with those reported in 1901 from the Thompson Yates Laboratory of Physiology.

This surprising delimitation of what had previously been considered a wide territory to a relatively narrow precentral strip, left, without definitely proven function, the large portion of Munk's sensori-motor fieldthe old "motor area" of others-lying posterior to the central fissure. Conjectures have naturally arisen in regard to the function of this field-one from which motion may be elicited by undue strength of ourrent-and the presumption that it is a sensory end-station has received support from certain histological studies (notably those of Alfred W. Campbell) as well as from a number of carefully observed clinical cases. ${ }^{2}$

1 Presented at a meeting of the American Physiological Society, December 90, 1908.

2 It would thus appear, as is so often the case with disputed points, that there is un element of truth on both sides; for the original " motor ares," embracing the convolutions on each eide of the central fissure, appears to be sensori-motor, though not in Munk's senso -namely, with coinciding or superimposed afferent and efferent stations.

There can be no doubt bat that lesions of the gyrus precentralis can lead to motor disturb. ances without recognizable sensory lose; on the contrary, a lesion limited to the gyrus postcentralis will almost inevitably inbibit normal free movements. I have seen a number of 
The experimental study of sensation in animals, being linited to the interpretation of reflex actions, is most difficult and unsatisfactory. Needless to say, it is a matter which requires on the part of the subject not only an analysis of his sensory impressions, but their transference by descriptive speech to the observer, who in turn must interpret this description; for at best it is difficult to find words precisely indicative of many forms of sensation.

Owing to the impossibility of this necessary communication the making of observations on the sensory cortex of unanæsthetized animals would be as futile as during a state of narcosis. This, however, is not the case with man.

A year ago I reported, with Dr. H. M. Thomas, a surgical experience ${ }^{1}$ in which at a second-stage operation an osteoplastic flap, previously made, was re-elevated, the dura reflected, the cortex incised, and a subcortical cystic tumour removed from the postcentral field of an unanæsthetized patient. The procedure, with the exception of accidental dragging upon the dura, and the final reclosure of the scalp, not only occasioned no discomfort, but was attended on the part of the patient by a lively and helpful interest in the performance. He even informed us of a focal sensory attack which occurred early in the proceedings, evidently incited by the preliminary palpation of the exposed brain. At the first session of the operation, under anæsthesia, the motor cortex had been electricallydelineated, and we greatly regretted that after safely concluding the second stage we had not taken the occasion to test with the faradic current the postcentral areas. ${ }^{2}$

instunces of this. Thus, in a patient with a glioma of the postcentral gyrus, partial hemi. anæsthesia was accompanied by marked motor disturbances, but no degeneration of the pyramidal tract was demonstrable at autopsy; and, further, an operative case witb prompt restoration of normal motility after the evacuation of a postcentral, subcortical clot in a patient presumed to be hemiplegic, has been deteiled in my Carpenter Lecture for 1906 (New York Medical Jon rnal, January 26, 1907).

On a few occrsions, in cases of focal epilepsy, characterized by a definite sensory aura in face or band, I have extirpated a seginent of the postcentral convolution corresponding witb the level of the precentral strip, from which movement in the part concerned was elioited by cortical atimulation at the time of operation. Occasionally, though not nlways, a certain degree of hypercesthesia bess subsequently ben demonstrable in the expected fleld; but this has always been a transient affair, clearing up in large part rapidly just as does a motor palsy resulting from the extirpstion of a small segment of the precentral gyrus.

These observations have never been such as to justify definite pbysiological conclusions, for the extirpated eegment has usually been small-a cubio centimetre of tissue possibly-and the deeper corter lining the wall of the flasure must often bave remained intact.

' "Removal of a Sabeortical Cystic Tumour at a Second-stage Operation without An asthesia," Journal of the American Medical Association, 1908, vol. i., p. 847.

2 A footnote describing a single, and the flrst experience in this direction, accompanied the article referred to above. In making a subtemporal defeot for purposes of decompression in a case of brain tumour-a procedure usually concluded under anestbesia at one sitting-it was necessary, owing to some meningeal bleeding, not easily cheoked, to postpone the opening of the durn to a second session. This was accomplished without an auresthetic, and the exposed temporal lobe was stimulated, giving the patient certain definite auditory impressions which he claarly distinguished from the "buzz" of the vibrator. 
In the past year, however, the exigencies of two surgical cases have given us the looked-for opportunity of making further observations upon the sensory field. It is the purpose of this paper to record solely the physiological aspects of these observations, although their surgical aspects are possibly of even greater importance; for if it will be possible in the future to pick out with an electrode areas of the brain from which the sensory aura of a focal convulsion has originated, we shall have advanced a long way toward the possible operative localization of subcortical irritative lesions of the immediately postcentral field.

Case 1. History.-The patient, a sturdy and intelligent boy of 15 years of age, was referred by Dr. H. M. Thomas to the surgical service of the Johns Hopkins Hospital on June 16, 1908, suffering from "convulsive attacks originating in right hand and right side of face." At the time of onset, two years before admission, these seizures were limited to a subjective sensory disturbance, characterized at first by periods of numbness in the little finger of the right hand, and finally by what was described as a "funny feeling" in the arm-a "gone sensation."

These purely sensory attacks increased in frequency and severity, and finally were followed by a twitching of the little finger; then by contractions involving the whole arm, and more recently by an extension of the motor disturbances to the right side of the face.

Though he had never himself observed an involvement of the right leg, the more severe seizures which occurred after his admission to the hospital were accompanied by a definite convulsive movement in the lower extremity as well.

A typical attack, many of which were observed, was preceded for a period of possibly ten minutes by a feeling of weakness or "goneness" in the arm. This was followed by tingling, roughly confined to the ulnar cutaneous distribution of the hand; then the arm would suddenly flex and be drawn up above the head. At this stage of the seizure he could occasionally check further progress of the "march" by grasping the affected arm with the left hand, and by pushing it violently to his side. No loss of consciousness accompanied the attacks, and usually, though not always, he could be marle to speak during them. They were attended by a sensation of appreliension, but never by one of pain.

In the most severe seizures he would be drawn to the floor by an arching of the body to the right, with convulsive movements of the right side; but even in these attacks he was never known completely to lose consciousness. A distinct though transient weakness in the arm with irregular atrxic movements usually followed the attacks.

Beyond the presence of an incipient choked disc (hyperwmic stage), and a slight disturbance of pain and temperature sense over the postaxial surface of the hand and forearm, the physical examination was practically negative. 
With the presumption that some organic lesion, possibly a tumour of the postcential territory, would be found, an exploration was advised.

Operation (first stage, June 16, 1908.)-An area of the left hemisphere, as shown in the accompanying sketch (fig. 1), was exposed by the usual osteoplastic craniotomy. The hemisphere was found under slightly increased tension, but no ubnormality was apparent.

Owing to the characteristic configuration of the central fissure, the landmarks of the exposed part of the hemisphere were perfectly clear, but the situntion of the precentral gyrus was further assured by unipolar stimulation which elicited the usual shurp movements in wrist, in elbow, in shoulder and in fnce. The only subcortical investigation at this time was limited to the explorn-

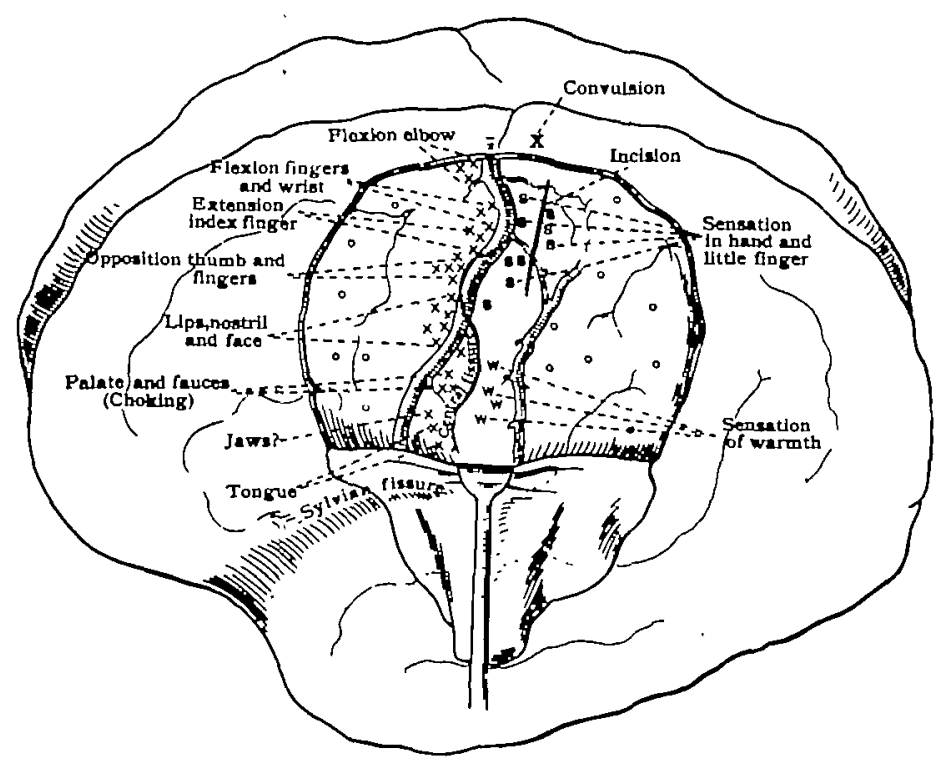

FIG. 1.

tory puncture of the postcentral gyrus in the vain hope that a cyst might thus be encountered. The wound was then reclosed.

The boy's distressing seizures continued unabated, until finally it was determined that some more radical attempt should be made to disclose, if possible, their source of origin. It was hoped that if the cortex could be stimulated while the patient was conscious; we might thus obtain some evidence of the situation of the presumed lesion sufficient to justify a more extensive subcortical explorntion.

Operation (second staye, July 6, 1908).- - After a preliminary injection of $\frac{1}{8} \mathrm{gr}$. of morphin, and under primary anæsthesia by chloroform, the edges of 
the former incision were newly separated and the original bone-flap quickly re-elevated. The patient promptly regained complete consciousness, and while in this state, without occasioning discomfort, the dura was divided in the line of the previous closure, and the membrane was again reflected.

Cortical stimulation.-The following electrical observations were then made. The same movements as before were easily elicited with a mild faradic current from the precentral strip. These motor responses in detail and in the order in which they were obtained from the areas shown in the accompanying sketch

- (fig. 1) consisted of :-

(1) An opposing movement of thumb and fingers from points opposite to the unmistakable genu.

(2) Somewhat higher up, extension of the index finger.

(3) Still higher flexion of the fingers, which carried over to flexion of the wrist.

(4) At the upper margin of the exposed field, flexion of the elbow.

(5) Below the evident middle genu the movements, which required a somewhat stronger current, consisted of contraction of the side of the fuce.

(6) Movements of palate and fauces, a curious choking sensation being occasioned whenever they were obtained; and

(7) From the very lowest exposed part of the strip movements of the tongue occurred, and the patient experienced a sensation of movement in the lower jaw, though this was not certified by the observers.

It is to bo noted that the subject was perfectly aware of these movements, and in the attempt to express the sense of feeling which was occrsioned by them he said that it was the same sort of a drawing sensation uppreciated when the muscles are "pulled" by peripheral stimulation-a familiar experience for him, owing to a long course of electrical treatment before his admission to the hospital. The movements, in other words, gave him the sensation of actire muscular contraction alone.

Posterior to the central fissure, over an area roughly corresponding with the upper two-thirds of the exposed postcentral convolution (fig. 1), stimulntion, with the same strength of current used to elicit the motor responses from the precentral gyrus, led to a sensation in the hand, arm and little finger-chiefly in the little finger-which was said to be exactly akin to the sensation that inaugurated his attacks. Stimulation lower down on the postcentral gyrus occasioned a sensation of warmth in the arm, but this was rather vague and indescribable.

It is to be noted that there were no painful sensations whatsoever, and further, that stimulation of the field in front of the motor strip and back of the postcentral convolution elicited no movements or sensations whatsoever, and the patient seemed to be unaware of the application of the electrode.

On elevating the upper edge of the durb at the point $X$ (fig. 1) and placing the electrode there, an attack precisely similar to his usual attacks, inaugurated by the same sensory disturbances and followed by the customary abduction of the arm and arching of the body to the side, was produced. This lasted but 
a few moments, and was not followed by a general convulsion nor by loss of consciousness."

In the light of these observations, n number of small vessels crossing the postcentral gyrus were doubly ligated and an exploratory excision $3.5 \mathrm{~cm}$. in length was then made between the ligatures (fig. 1) and carried down to a depth of about $2.5 \mathrm{~cm}$., unfortunately without disclosing any lesion. The procedure was practically bloodless, and the edges of the incised pia-arachnoid were subsequently approximated with three delicate split silk sutures. No sensation whatsoever accompanied the making of this deep exploration.

The dura was reclosed, the bone-flap replaced, and under a second primary chloroform anæs thesia the scalp was quickly resutured. ${ }^{2}$

Case 2. History. $-\Lambda \mathrm{n}$ intelligent man, aged 44 , began to suffer in August, 1907, with attacks of Jacksonian epilepsy of obscure origin. These attacks, beginning in and oiten limited to the right hand, were trifling at first, but soon became more numerous and more severe. They were usually preceded by an uncomfortable numb feeling in the palm of the hand, associated with a sense of weakness. This definite aura would sometimes fade away without further progress, bat more frequently would pass over into a motor attack. Oiten the definite motor seizures were inaugurated by a stinging sensation "like an electric shock," which would seem to ran down between the little and ring fingers.

The earliest effect upon movement would be an uncontrollable extension, particularly of the ring and little fingers, with abduction of the latter. In attacks of a somewhat more severe type a "drawing" sensation would pass up the arm, accompanied by flexion of the elbow, abduction of the shoulder, and

1 It is impossible to sny whether or not stimulation of the postcentral convolution at its upper and mesial extremity, and possibly over on the mesial surface of the hemisphere, would have given sensory impressions in the lower extremity. No sensation occurred in the face from stimulation of the postcentral convolution at a level below the middle genu; but it is here again conceivable that the earlier excitation of the presumed sensory arens for hand may have led to their over-excitability, with the possible masking of a lesser sensation that may have occurred in the bensory area for face.

It may be noted that the mesial edge of the dural opening was $2 \mathrm{~cm}$. from the mid-line; the exposed field measured $8 \frac{1}{2} \mathrm{~cm}$. in width, and $7 \mathrm{~cm}$. in a vertical direction.

I Though vot essentisl to the main purpose of this report, the postoperative results of this cortionl incision may deserve notice. It led to a slight sensory disturbance, limited to the ulaar side of the hand, little, and ring fingers. Tactual impressions over this ares were dulled; there was a slight degree of hypalgesin, particularly at the tip of the little finger; aud on the ulnar side of the forearm a temporary difficulty of distinguishing between beat and cold. There was subjective complaint also of the hands feeling tired. The steriognostic faculty was unaffected, but a loss of postural sense was demonstrable in the little finger.

The disturbnnce of afferent impressions was further evidenced by the slightly increased difflulty of performing fiuer movements, as in writing. The arm was a trifle stiff from an evident musoular hypertonicity, but muscular strength was unimpaired.' Some incoordination in movement, increased during the closure of the eyes, was appreciable. All of these postoperative disturbauces faded away in the course of a few weeks.

The surprising fact is to be noted that the corticnl lesion occasioned sensory disturbances which appeared to be linited to the postarial side of the arm, rather than in a distribution commonly supposed to chnracterize peripheral ancesthesias of cerebral origin-namely, of the "stocking" and "glove" type.

It is unnecessary to add that a possible extracrnnial source of origin for these sensory disturbances on the postaxial side of the limb, such as a cervical rib or other peripheral lesion, had been carefully eliminated. 
tilting of the head to the right. During the seizures, even of this degree of severity, though confused and unable to respond when questioned, he would not lose consciousness. On a few occasions, however-six in all- 2 severe general convulsion has occurred, in which the inaugural sensory disturbance has passed down the side of the body before consciousness was lost. ${ }^{1}$

His history otherwise and the physical examination were practically negative.

Operation (first stage, December 8, 1908). - The patient took his anmsthetic badly, and, owing to the attendant cyanosis, an unusual amount of blood was lost while elevating the large osteoplastic flap. It consequently was deemed best to postpone further measures to a second sitting, in the hope that with the patient unanasthetized the durn might be opened and the suspected lesion sought for by cortical stimulation in case its situation was not immediately apparent.

Operation (second stage, December 14, 1908).-After the administration of $\frac{1}{8} \mathrm{gr}$. of morphin and under primary anæsthesia from a few inhalations of chloroform, the original incision in the scalp was re-opened and the bone-flap re-elevated. The patient composedly and promptly recovered from the temporary narcotic effect of the drug. The dura was then opened in a line concentric with the incision in the scalp and bone and the membrane was reflected, no sensation of discomfort attending this procedure.

No abnormalities were evident on the surface of the exposed hemisphere, the topography of which was somewbat less clear than usual, owing to the presence of a large, single anastomotic vein (fig. 2), which was shown later, upon cortical stimulation, to overlie and thus obscure the greater part of an uncommonly straight Rolandic fissure.

Stimulation Observations.-Using a weak faradic current, ${ }^{2}$ of one-half the strength that had been found necessary to elicit distinctive movements from the cortex of a recent case under anæsthesia, the convolution in the middle of the exposed field, just posterior to the aforementioned vein, was lightly touched for the briefest moment of time with the tip of the coiled platinum-wire electrode (Sherrington pattern).

The patient, who had been informed of our object and who was enger to assist in every way in his power, immediately responded, saying that he experienced a sensation which he located in the index finger of the right hand. On further questioning he said it felt "as though someone had touched or stroked the finger." (This and the following observations were corroborated from time to time by repetitions of the stimulus.) From points lower down on the same gyrus, a similar sensation of "touching" or "stroking" was experienced, but from this lower area the feeling was located on the back of the hand.

'A number of the minor attaoks were observed and carefully described by Dr. H. M. Thomas, who had first seen the patient in consultation with Dr. J. M. T. Finney.

'The broad plate of the other pole in this, as in the former case, was applied to the thigh of the same side [left]. 
In order to be assured of the exact convolution from which these sensory responses occurred the gyrus lying anterior to the large vein was then touched about in the middle of the exposed field and opposite to what appeared to be a possible middle genu; a prompt flexion of the thumb into the palm of the hand followed this stimulus. The movement was unaccompanied by any sensation other than that of change of position of the thumb, which the patient promptly appreciated and described. In other words, there seemed to be no associated cutaneous sensation.

This latter observation was sufficient to certify the position of the fissura centralis (Rolandi), and although no further points on the precentral gyrus were subsequently stimulated, this same movement of the thumb was elicited is a control several times during the course of the later observations.

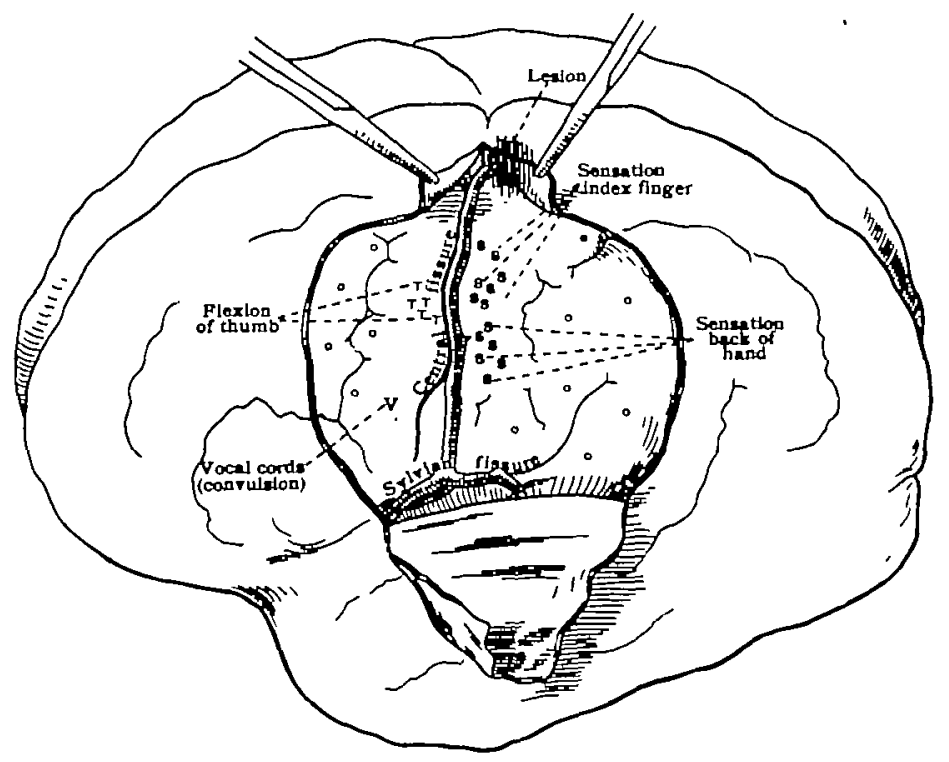

Fig. 2.

The precentral and postcentral gyri having thus been identified, the outlying convolutions were "coursed" in order to see whether any subjective sensations occurred from stimulation of other than the postcentral gyrus from which the sensory impressions had first been obtained. Thus the crests of all of the exposed convolutions, at about the points marked by small circles in the sketch (fig. 2), were stimulated, with the elicitation of no sensory and, needless to say, of no motor responses whatever. Occesionally, in order to be sure that the current was sufficient for reaction, the primary stimulation of the area giving sensation of the index finger and of that giving movement of the thumb was repeated.

In coursing the exposed portion of the hemisphere as described, the operator 
finally touched the point $V$, which immediately led to a curious, long-drawn, hoarse inspiratory sound, evidently due to closure of the glottis; this was followed by a general convulsion, the first observable evidence of which was a drawing-down of the right platysma and corner of the mouth, with depression of the chin and separation of the lips.'

Apprehensive lest a possible repetition of this unexpected convulsion might occur, and, from cyanosis and protrusion of the exposed cortex, occrsion some damage, the operator desisted from further stimulation, although the exact area which occasioned the inaugural sensation of the patient's seizures had not been located.

At this juncture the administration of chloroform was resumed, and, under the anæsthetic, the upper and mesial edge of the postcentral convolution was more fully exposed by ronguering awny a further portion of the skull at the upper edge of the cranial opening. This permitted an upward enlargement of the dural opening, as shown in fig. 2, and a lesion of the postcentral convolution, which was thought to be irremovable, was thus brought into view.

\section{CoMment.}

These observations seemingly add confurmation to the view which ascribes a sensory function to the gyrus centralis posterior, but beyond this I shall make no endeavour to interpret their significance. Just what relation the afferent impulse bears to a motor response ; just how stimulation of this supposedly sensory field is interpreted by the subject as sensoly; why the motor response from stimulation of the gyrus centralis anterior occurs without any primary afferent impression, but only with the secondary consciousness of the movement-these and many other questions that arise are matter's rather for the psychologist.

It is, perhaps, noteworthy that the sensations which were obtained were limited entirely to the hand and arm; and whether this means that there is an especially wide cortical representation for afferent impulses from this region, or whether these patients, owing to the characteristics of their seizures, possessed sensory centres from hand and arm which were unduly excitable, is difficult to tell, and will need further elucidation.

It is noticeable, in the last case, that the lesion which occasioned the

'It has seemed to me probable, from other experiences in the past, that the cortex in cnses of epilepsy is more excitable than the cortex of non-epileptics; in the former it is traditionally easy, in fact, to occasion, even under anesthesia, a Jacksonian fit which may run into a general convulsion. No apprehension, however, of this possible consequence of stimulation was felt in this particular patient, as such a weak current was being used. The numerous, rapidly executed and repeated stimulations of the seemingly non-excitable arens of the hemisphere may possibly have had an accumulative effect, so that when again approaching the lower part of the precentral convolution (Horsley's vocal-cord centre) the response to the stimulus was much more pronounced than was anticipated or was desirable. 
seizures was found to lie higher on the postcentral gyrus than the areas. which had been stimulated, so that presumably responses from the hand would have been brought about by stimulation there, just as they had been from a lower level of the convolution. It is possible, too, that the patient's attention in each case may have been so concentrated upon the affected hand that sensory impressions arising from leg, body and face passed unobserved. The fact that the sensations in the first case were precisely the same as those characterizing the aura of the patients' seizures would lend support to this view; but it was otherwise with the second case, for in this patient, though sensations occurred in the hand, they were other than those characterizing the inaugural stage of the fit and occured over different skin fields.

\section{SUMmarY.}

Two patients, afflicted with epileptic attacks inangurated in each instance by a sensory aura in the right hand, offered unusual opportunities, as related, for cortical stimulation while in a conscious state during a "second-stage" operation.

In both of them the situation of the central fissure was determined by obtaining characteristic motor responses from the precentral gyrus, these motor responses being attended by no sensation other than that of the forced change of position which accompanies similar movements elicited by stimulation of a peripheral nerve.

On the other hand, in both of these patients stimulation of the postcentral convolution gave definite sensory impressions which were likened in one case to a sensation of numbness, and in the other to definite tactual impulses.

In both of the patients, furthermore, stimulation of the outlying convolutions gave no response whatsoever, either of a subjective sensation or of active movement. 